1 Independent Panel for Pandemic Preparedness and Response Secretariat, Global Health Campus, Geneva, Switzerland

2 School of Public Health, Boston University, USA

3 Institute of Health Policy, Management and Evaluation, University of Toronto, Canada

4 Saw Swee Hock School of Public Health, National University of Singapore, Singapore

5 Department of Global Health and Development, London School of Hygiene and Tropical Medicine, UK

6 Faculty of Medical and Health Sciences, University of Auckland, New Zealand

7 Independent Panel for Pandemic Preparedness and Response

Correspondence to: Salma M Abdalla abdallas@bu.edu

Cite this as: $B M J$ 2021;375:e067487 http://dx.doi.org/10.1136/bmi-2021-067487 Published: 29 November 2021

COVID-19 PREPAREDNESS AND RESPONSE: IMPLICATIONS FOR FUTURE PANDEMICS

\title{
Investing in trust and community resilience: lessons from the early months of the first digital pandemic
}

Salma M Abdalla and colleagues examine how an atmosphere of misinformation, disinformation, and erosion of trust shaped the early response to covid-19 on both global and national levels

Salma M Abdalla, ${ }^{1,2}$ Shaffi Fazaludeen Koya, ${ }^{2}$ Margaret Jamieson, ${ }^{3}$ Monica Verma, ${ }^{4}$ Victoria Haldane, ${ }^{3}$ Anne-Sophie Jung, ${ }^{5}$ Sudhvir Singh, ${ }^{1,6}$ Anders Nordström, ${ }^{1}$ Thoraya Obaid, ${ }^{7}$ Helena Legido-Quigley, ${ }^{1,4,5}$ Christine McNab

The covid-19 pandemic has underscored the importance of risk communication and community engagement (RCCE) in preparedness and response efforts to health threats. It has illustrated the realities of a world that is digitally interconnected, a world in which misinformation can spread more easily than ever before and contribute to the erosion of trust. Given these realities, this analysis aims to highlight the need to invest in community resilience as a core component of preparedness and response for health threats.

\section{Covid-19 in context: the first digital pandemic}

Communication during disease outbreaks, particularly for novel pathogens, is challenging. Rapidly evolving statistics, health information, recommended public health measures, and best practices must be communicated consistently and transparently, and tailored for a wide variety of audiences. These audiences often have differing languages and dialects, cultural and religious beliefs, and access to information technology.

These challenges have been magnified during the current pandemic. Covid-19 has laid bare existing societal limitations at a time when people have the ability to share and receive information globally at unprecedented speeds.

Information about what was later identified as SARS-CoV-2 spread faster globally than the virus itself. On 31 December 2019 an online Chinese news outlet published a Wuhan Municipal Health Commission's internal notice concerning cases of pneumonia of unknown origin. Within three hours, ProMed-mail (an internet based infectious diseases outbreak reporting service) translated and published the article online, 40 minutes before the Wuhan Municipal Health Commission issued a public bulletin.

By 1 January 2020, mainstream news outlets were reporting on the outbreak, and the World Health Organization took the unprecedented move of announcing a new outbreak through Twitter on 4 January 2020. The demands for information were justifiable, unrelenting, and insatiable. In the first six months of the pandemic, social media and online websites recorded 270 billion shares and views of information about covid-19. ${ }^{2}$

\section{Widespread misinformation and disinformation}

The internet and social media helped quickly disseminate information about SARS-CoV-2. However, they also spread confusion, especially with rapidly changing, and sometimes conflicting, evolving scientific knowledge about the virus, the damage it could do, and the measures people should take to protect themselves. Information splintered through a prism of languages, social media feeds, biases, geopolitical tensions, fears, and varying levels of health literacy.

Misinformation, and worse, disinformation became an acute problem when prominent political leaders used social media to question the prevailing science and supported unverified treatments (box 1). By 2 February 2020, WHO announced that the world was experiencing not only a pandemic but also an infodemic that made it more difficult for people to find trustworthy guidance and sources.

Box 1: Efforts to tackle covid-19 misinformation and disinformation

In an attempt to tackle the evolving infodemic in early 2020, many countries, including Singapore, Thailand, and Vietnam, passed legislation against online misinformation.

Social media corporations also struggled to address misinformation on their platforms. By March 2021, YouTube had removed around 800000 videos containing covid-19 misinformation, of which 30000 were focused on vaccines. ${ }^{3}$ Still, it was only in February 2021 that many platforms banned sharing anti-vaccination information. In particular, "end-to-end" encrypted applications such as WhatsApp, which is a popular application in many low and middle income countries, are a significant source of misinformation. These platforms are challenging to monitor, and by extension counter, because the sender of the information may not be known, and information can be forwarded instantly and easily to large numbers of people.

\section{The fall out: erosion of trust}

Another contextual factor that affected the trajectory of the covid-19 pandemic is the level of trust 
communities have in their institutions. Trust is a reflection of positive perception about the actions of an individual or an organisation that shapes behaviour. Garnering and maintaining trust is important to foster citizens' cooperation with government officials and institutions. 45

A key pillar of effective implementation of public health measures is thus earning and maintaining the trust of communities. In a disease outbreak, trust in three types of actors is relevant to required behavioural change: the government that imposes the required changes, fellow citizens whose cooperation is needed for individual efforts to be effective, and scientists who provide the evidence for change. ${ }^{6}$ In addition, the degree to which people feel marginalised before an outbreak plays a part in their trust of authorities.

Communities that feel isolated because of racial prejudice or neglect, for example, will be more reluctant to follow public health advice and tend to question health messages. ${ }^{78}$

The central role of trust was clear during the 2014-2015 Ebola outbreak in west Africa. A large representative survey in Liberia at the time showed that people who expressed a lack of trust in government ignored precautions against Ebola in their homes and were reluctant to follow Ebola control policies. ${ }^{9}$

The covid-19 pandemic occurred at a time of low levels of trust in governments in many countries. For example, at the beginning of 2020 a survey of 34 ooo people in 28 countries showed that only $42 \%$ of participants had confidence that government leaders could tackle the challenges of the day. By 2021, trust in governments had decreased even further. ${ }^{10}$

This low level of trust can potentially be attributed to at least four factors. Firstly, early communication efforts often failed to underscore that covid-19 science would evolve rapidly. This later contributed to resistance to revised public health directives and guidance. Secondly, increasing polarisation of public views within some countries fed debates about the validity of science, and led to an increase in misinformation and disinformation about covid-19 prevention and treatment.

Thirdly, geopolitical tensions that led some national leaders to attack United Nations agencies, especially WHO, impacted the public's trust in these institutions. This left international institutions sometimes struggling to navigate the politics while communicating in ways that maintained credibility. Fourthly, a significant challenge during the pandemic has been the balance between effectively tackling the virus and preserving human rights. A number of governments have exploited emergency laws to clamp down on civil liberties and attack political opponents, thus not only eroding trust but putting citizens at risks superseding the health threats of covid-19. ${ }^{11} 12$

Ultimately, public trust and the covid-19 infodemic are interconnected. The spread of misinformation and disinformation is a manifestation of a deeper lack of trust in governments and institutions. ${ }^{13}$ The spread of the covid-19 infodemic reflects people's expressed desire to be informed, consulted, and included in the response plans and their execution.

\section{RCCE efforts during the covid-19 pandemic}

The importance of sustained investment in communication and communities is supported by ample evidence. The term community generally refers to groups with a common set of social relationships that formulate a shared identity among members. In this analysis, we often use the term communities to refer to population sub-groups within a country. During the early months of the pandemic, many communities across the world organised themselves to tackle the virus and help those most affected by its social and economic impact. While many occurred organically, organised efforts, especially by governments, provide lessons to improve future communication and community engagement efforts (box 2).

Box 2: Examples of emerging themes from successful community engagement efforts during covid-19 ${ }^{14}$

Effective mobilisation of existing community structures, resources, trusted organisations, and knowledge bases

- Local health authorities in northwest Syria adopted a decade old polio vaccination mobilisation system of local volunteers, and a medical network across the border in Turkey to raise covid-19 awareness, refer patients, and train health workers

- Self-help groups of women in India were entrusted to run community kitchens for quarantined people

- Local women associations in Yemen and international agencies trained women in a rural district to produce masks and personal protective equipment

Institutionalising risk communication and community engagement in preparedness and response

- Thailand had a well designed plan with a clear command structure and defined responsibilities; an all-hazards risk communication plan; and a focused budget. They used this early on in the pandemic to introduce a "stay home, stop the virus, for our nation" campaign nationally and across its 76 provinces using the existing trusted network of Village Health volunteers

- South Korea's Centre for Disease Control and Prevention Office of Communication acted as a single focal point of communication of all technical and scientific information, and began sending messages about covid-19 through multiple channels before the first case was reported in the country

Developing and investing in innovative uses of technology and social media channels

- "Route maps," digital tools in India for contact tracing

- Testing a site locator mobile app in the United States

Promoting a culture of civic mindedness

- China, Japan, and Taiwan emphasised "consideration of others" in public health messages to promote mask use

Clear, consistent, transparent, and action oriented messaging

- Leaders in Singapore, Ireland, and New Zealand consistently delivered updates, including transparent information about what was known and not known about covid-19

Yet overall, RCCE are too often treated as supplementary activities during emergencies rather than core components of preparedness and response. As a result, neither area typically receives adequate investment. Moreover, community engagement is often framed as parallel to, or even a sub-component of, risk communication efforts, rather than an overarching goal. These challenges have remained during the covid-19 pandemic (box 3).

Box 3: Investment in risk communication and community engagement (RCCE) before and during the early months of the covid-19 pandemic

Internationally, RCCE has generally been dependent on waves of unsustainable project based funding linked to emerging public health emergencies such as severe acute respiratory syndrome (SARS), influenza, or Ebola. For example, despite known RCCE challenges arising from the 2014-2015 Ebola response, the Collective Service (a partnership bringing together the strengths of the International Federation of Red Cross and Red Crescent Societies, United Nations Children's Fund, and WHO) was established only in June 2020 once covid-19 had spread worldwide. It was initially funded by an external six month grant. Further, these earlier 
efforts were often mainly focused on risk communication rather than community engagement.

Nationally, a pre-covid-19 assessment of countries' preparedness and response abilities as per the International Health Regulations highlighted the low prioritisation for RCCE among WHO member countries. In particular, indicator 5.4 ("community engagement with the affected communities") had the second lowest average score of the five indicators relevant to RCCE. ${ }^{15}$ Moreover, despite increasing political and operational commitment to community engagement, funding for both is often limited, supporting ad hoc reactionary interventions rather than sustained action. ${ }^{14}$ However, as the pandemic progressed, governments began prioritising RCCE efforts. In April 2020 , only about $36 \%$ of countries reported having an RCCE plan, but that number increased to $90 \%$ by October 2020.

Importantly, these numbers do not necessarily reflect the reality of RCCE efforts within countries, as the definition of RCCE varies by country. A review for the Independent Panel for Pandemic Preparedness and Response showed the varying levels of RCCE efforts among 28 countries for the first year of the pandemic analysed. Overall, apart from a few, countries lacked formal community engagement plans as part of their response efforts. ${ }^{16}$

\section{Lessons to invest in RCCE as a true pillar of preparedness and response}

The ongoing pandemic has shown the need to centre communities in preparedness and response efforts more than ever before. It highlights the limitations of the current approach to RCCE (box 3) as ancillary components of preparedness and response and the need to systematically invest in RCCE. Four lessons have emerged thus far.

\section{Effective risk communication strengthens community engagement}

Effective risk communication provides communities with actionable, timely, and honest information online and offline, delivered by trusted leaders. It also builds trust by establishing open communication channels with communities, incorporating their concerns and feedback to improve communication.

Social media can benefit risk communication, but it can also be destructive and foster social polarisation. Broken communication systems lead to confusion on the ground and sow the seeds of misinformation and mistrust, especially during health threats with evolving knowledge. As such, risk communication should clearly reflect the evolving nature of science.

\section{Community ownership is key}

Risk communication is necessary but insufficient on its own. A community sense of ownership and involvement is an important success factor in tackling health threats. Communities, particularly marginalised communities, must be early partners in all stages of the design, planning, implementation, and assessment of preparedness and response. This requires government transparency and accountability, and a sincere appreciation and recognition of the importance of community engagement by authorities.

\section{Establishing and maintaining trust is an ongoing process}

Community engagement is not a one-time effort and cannot "happen" in times of crisis. Successful community engagement efforts have to be maintained before, during, and after disease outbreaks, and require clear structures and sustained funding. This is how governments can foster community cooperation during times of fear, vulnerability, and uncertainty. Without sustained investment, particularly in marginalised communities, engagement during outbreaks risks community resistance rather than cooperation.

These lessons are perhaps not new, but the experiences of the covid-19 pandemic must serve as clear evidence for urgent sustained investment, particularly in an increasingly polarised and digitally interconnected world. Embracing a community resilience framework in preparedness and response efforts offers a potential pathway forward.

\section{Case for adopting a community resilience framework}

Community resilience refers to the ability of a community to both mitigate adverse effects and recover from a disaster. Adopting a community resilience framework has been helpful for tackling large disruptions historically-for example, natural disasters. ${ }^{17}$ In particular, such an approach can help tackle some of the challenges identified in RCCE efforts during the covid-19 pandemic. Adding to other voices, we argue that such a framework for action can be adapted to tackle disease outbreaks. ${ }^{18}$ Incorporating a community resilience approach to pandemic preparedness and response presents an opportunity to shift from the traditional efforts that focus mainly on immediate actions during pandemics to investment before, during, and after a pandemic.

RCCE strategies are integral aspects of such an approach. A community resilience framework requires investment in effective risk communication through the ongoing provision of public information on preparedness, risks, and relevant actions before, during, and after a large societal disruption. Information must be delivered by credible sources early and transparently. It must include a focus on communicating what is known and not yet known, and a clear signal that public health advice may change as the science evolves. Beyond risk communication directly related to the virus, Yip et al recently argued that such messaging should emphasise cooperation, compassion, and kindness to promote a culture of civic mindedness. ${ }^{18}$

A community resilience approach also encourages participatory decision making. It requires the integration and involvement of communities through partnerships between governments and community based entities to co-design interventions that deal with the specific needs of local communities. Adopting this component of the community resilience framework can help foster and maintain trust with communities during an emerging disease outbreak.

Importantly, a core component of the community resilience approach is that governments invest in the social and economic wellbeing of communities before and during emergencies. Community resilience is strongly impacted by existing levels of social protections. Covid-19 brought into focus the grave existing socioeconomic inequities that fuelled the pandemic in many countries and were then exacerbated by the pandemic, highlighting the need to invest in social and economic protections.

Resilient communities are more prepared to mitigate the adverse effects and recover from large societal disruptions. These are not future potentials but are known threats: covid-19 continues to be a health threat, another novel virus could emerge at any time, and the impact of climate change is already causing disruption.

\section{Key messages}

- The unprecedented ability and speed to share information have marked the covid-19 pandemic. Social media enabled an atmosphere of misinformation that built on, and led to, a corrosion of trust in institutions. 
- Despite ample evidence of its importance, RCCE efforts are often not institutionalised or funded as core components of pandemic preparedness and response.

- Embracing a community resilience approach to pandemic preparedness and response requires a paradigm shift from the traditional efforts that mainly focus on reactive top-down actions to investment in community engagement before, during, and in the aftermath of health threats.

Contributors and sources:SMA, SFK, MJ, MV, and CM conceived and designed the manuscript. SMA, SFK, MJ and MV drafted the manuscript with inputs from all authors. All authors contributed to revising the manuscript and approved the final version. This analysis was part of the work commissioned by the Independent Panel for Pandemic Preparedness and Response, which reviewed the national responses of 28 countries in addition to the global response to covid-19. The analysis is separate from the panel's final report and has been facilitated by the panel secretariat. The secretariat is independent and impartial. The views expressed in this work are solely those of the authors and do not represent the views of the panel.

Competing interests: We have read and understood BMJ policy on declaration of interests and have no interests to declare.

Provenance and peer review: Commissioned; externally peer reviewed.

This collection of articles was proposed by The Independent Panel for Pandemic Preparedness and Response. Open access fees were funded by WHO and Singapore's National Medical Research Council (NMRC/CG/CO26/2017_NUHS). The BMJcommissioned, peer reviewed, edited, and made the decision to publish these articles. Kamran Abbasi was the lead editor for The BMJ.

1 Madoff LC, Madoff LC. ProMED-mail: an early warning system for emerging diseases. Clin Infect Dis 2004;39:227-32. doi: 10.1086/422003. pmid: 15307032

2 United Nations. New Africa alliance aims to tackle deadly COVID 'infodemic'. UN News 2020 https://news.un.org/en/story/2020/12/1079222

3 YouTube deletes 30,000 vaccine misinfo videos. BBC News 2021 Mar 12. https://www.bbc.com/news/technology-56372184

4 Bounding KE, Easton D. A systems analysis of political life. Behav Sci 1968;13:147-9doi: 10.1002/bs.3830130208.

5 Dalton RJ. The social transformation of trust in government. Int Rev Sociol 2005;15:133-54 doi: 10.1080/03906700500038819.

6 Baron J. Trust: beliefs and morality. In: Ben-Ner A, Putterman L, eds. Economics, values, and organization. Cambridge University Press, 1998: 408-18, doi: 10.1017/CBO9781139174855.017.

7 Quinn S, Jamison A, Musa D, Hilyard K, Freimuth V. Exploring the continuum of vaccine hesitancy between African American and white adults: results of a qualitative study. PLoS Curr 2016:8:ecurrents.outbreaks.3e4a5ea39d8620494e2a2c874a3c4201. doi: 10.1371/currents.outbreaks.3e4a5ea39d8620494e2a2c874a3c4201

8 King C, Leask J. The impact of a vaccine scare on parental views, trust and information needs: a qualitative study in Sydney, Australia. BMC Public Health 2017;17:106. doi: 10.1186/s12889-017-4032-2. pmid: 28114986

9 Blair RA, Morse BS, Tsai LL. Public health and public trust: Survey evidence from the Ebola Virus Disease epidemic in Liberia. Soc Sci Med 2017;172:89-97. doi: 10.1016/.socscimed.2016.11.016. pmid: 27914936

102021 Edelman trust barometer. https://www.edelman.com/trust/2021-trust-barometer

11 Covid: How the war on the virus attacked freedom in Asia. BBC News 2021 Jan 3. https://www.bbc.com/news/world-asia-55362461

12 Nay O. Can a virus undermine human rights? Lancet Public Health 2020;5:e238-9. doi: 10.1016/S2468-2667(20)30092-X. pmid: 32325013

13 Han Q, Zheng B, Cristea M, etalPsyCorona Collaboration. Trust in government regarding COVID-19 and its associations with preventive health behaviour and prosocial behaviour during the pandemic a cross-sectional and longitudinal study. Psychol Med 2021:1-11. doi: 10.1017/S0033291721001306. pmid: 33769242

14 Independent Panel. Centering communities in pandemic preparedness and response. 2021. https://theindependentpanel.org/mainreport/

15 Global Preparedness Monitoring Board. From words to action: Towards a community-centred approach to preparedness and response in health emergencies. 2021.

https://www.gpmb.org/annual-reports/overview/item/from-words-to-action-towards-a-community-centred-approach-to-preparedness-and-response-in-health-emergencies

16 Haldane V, De Foo C, Abdalla SM, etal. Health systems resilience in managing the COVID-19 pandemic: lessons from 28 countries. Nat Med 2021;27:964-80. doi: 10.1038/s41591-021-01381-y. pmid: 34002090

17 Koliou M, van de Lindt JW, McAllister TP, Ellingwood BR, Dillard M, Cutler H. State of the research in community resilience: progress and challenges. Sustain Resilient Infrastruct 2018;№ Volume:131-51. doi: 10.1080/23789689.2017.1418547. pmid: 31080883

18 Yip W, Ge L, Ho AHY, Heng BH, Tan WS. Building community resilience beyond COVID-19: The Singapore way. Lancet Reg Health West Pac 2021;7:100091

doi: 10.1016/.lanwpc.2020.100091. pmid: 33521745
This is an Open Access article distributed under the terms of the Creative Commons Attribution IGO License (https://creativecommons.org/licenses/by-nc/3.0/igo/), which permits use, distribution, and reproduction for non-commercial purposes in any medium, provided the original work is properly cited. 